Ciencia y Educación, Vol. 5, No. 1, enero-abril, 2021

ISSN (impreso): 2613-8794・ISSN (en línea): 2613-8808

DOI: https://doi.org/10.22206/cyed.2021.v5i1.pp25-40

\title{
Investigación educativa en la formación del profesorado: el caso de la Universidad de La Serena
}

\author{
Educational research in teacher training: the case of the \\ University of La Serena
}

Haylen Perines ${ }^{\mathrm{a}}$ ORCID: 0000-0002-7020-1014

Recibido: 25/03/2020 • Aprobado: 26/05/2020

Cómo citar: Perines, H. (2021). Investigación educativa en la formación del profesorado: el caso de la Universidad de La Serena. Ciencia y Educación, 5(1), 25-40. https://doi.org/10.22206/cyed.2021.v5i1.pp25-40

\begin{abstract}
Resumen
El objetivo del presente trabajo es determinar la presencia de la investigación educativa en los planes y programas de formación de las carreras de pedagogía de la Universidad de La Serena (Chile). Es el primer estudio en Chile que aborda esta temática, lo que significa un aporte importante a la literatura vigente. Se realizó un estudio cualitativo a través de un análisis documental de los once planes y programas de estudio antiguos y de los renovados para el año 2019, utilizando como referencia elementos del análisis del discurso propuestos por Van Dijk (1980). Los resultados apuntan a que la investigación educativa tiene una presencia escasa dentro de los programas antiguos. Los planes y programas renovados, en cambio, tienen una inclusión más intencionada y organizada de ella. Entre las conclusiones se menciona la importancia de llevar a cabo cambios progresivos y graduales respecto a la forma de abordar la investigación educativa en la formación de profesorado.
\end{abstract}

Palabras clave: investigación; pedagogía; formación de profesores; educación superior; programas.

\begin{abstract}
The objective of this research is to determine the presence of educational research in the plans and training programs of the pedagogy careers of the University of La Serena (Chile). It is the first study in Chile that addresses this topic, which means an important contribution to current literature. A documentary analysis of the old and renewed study plans and programs for 2019 was carried out using as reference elements of the discourse analysis proposed by Van Dijk (1980). The results suggest that educational research has a scarce presence within the old programs. Renewed plans and programs, on the other hand, have a more deliberate and organized inclusion of it. Among the conclusions, the importance of carrying out a profound and substantial change regarding the way of approaching educational research in teacher training is mentioned.
\end{abstract}

Keywords: research; pedagogy; teacher training; higher education; programs.

a Instituto Multidisciplinario de Ciencia y Tecnología. Universidad de La Serena, Chile. Correo-e: haylen.perines@userena.cl 


\section{Introducción}

"Formar profesionales que utilicen los métodos de la ciencia para transformar la realidad es una necesidad"; esta cita, extraída del estudio de Rubio et al. (2018, p. 336) refleja la orientación principal de este artículo y pone en evidencia lo que se abordará: la importancia de una formación profesional que considere los métodos científicos en sus programas como parte de una nueva perspectiva sobre la realidad educativa.

Cabe preguntarse por qué la investigación educativa debería tener mayor presencia e importancia en los programas de formación de profesorado. La literatura vigente ha evidenciado una serie de beneficios que las actividades de investigación educativa tienen para los estudiantes de las carreras de pedagogía. Por ejemplo, gracias a ella los estudiantes pueden mejorar sus competencias profesionales (Guilbert et al., 2015), pueden aprender a interpretar la investigación de otros (Ion, 2014) y pueden tomar mayor conciencia de su propio aprendizaje (Todd, Bannister y Clegg, 2004). De acuerdo con Goodyear y Zenios (2007) la participación de los estudiantes en la investigación educativa les permite desarrollar su capacidad de comprender y participar en el proceso de producción de conocimiento.

Por otra parte, Levy y Petrulis (2012) consideran que las actividades de investigación educativa permiten que los estudiantes tengan una mayor autoconfianza e independencia en el aprendizaje y un mejor desarrollo de habilidades de pensamiento, como el análisis o la resolución de problemas. Todos estos beneficios posibilitan que haya autores para los cuales la investigación educativa debe ser introducida desde los primeros años de estudio (p. ej., van der Linden et al., 2012).

Cuando los estudiantes de pedagogía inician su formación profesional comienzan a recibir una serie de contenidos y habilidades pertinentes a su profesión. Existen asignaturas relativas a la disciplina elegida por el estudiante: lenguaje, matemáticas, ciencias, artes, las que se complementan con las propias de la formación como docentes. En estas últimas se pueden encontrar una variedad de saberes, que en general incluyen contenidos sobre currículo, evaluación, metodologías de enseñanza y prácticas. Sin embargo, entre estas asignaturas la investigación educativa no figura como un elemento prioritario, no tiene la presencia e importancia que debería en la formación de profesores y parece estar "invisibilizada” (Caín y Allan, 2017).

La escasa formación en investigación de los futuros docentes radica fundamentalmente en la presencia insuficiente de elementos de investigación en la organización curricular de las carreras de pregrado y en la débil importancia que se le otorga al tema en el transcurso de ellas (Perines, 2018; Perines y Campaña, 2019). En este contexto, el presente estudio tiene por objetivo determinar la presencia de la investigación educativa en los planes y programas de formación y en el perfil de egreso de las carreras de pedagogía de la Universidad de La Serena (ULS-Chile), teniendo en cuenta el proceso de renovación curricular que están viviendo actualmente las once carreras de pedagogías de la ULS.

Cabe mencionar que este trabajo muestra los primeros resultados de la investigación "Presencia e importancia de la investigación educativa en la formación pedagógica y su valoración por estudiantes y docentes" financiada por ANID (Agencia Nacional de Investigación y Desarrollo del Gobierno de Chile), a través del proyecto Fondecyt de iniciación, №11180227 adjudicado por la autora.

\subsection{Marco teórico}

La realidad en Chile es que en muchos planes de estudio de pedagogía existen escasas asignaturas relacionadas directamente con la investigación, las que, en caso de existir, se llevan a cabo en los semestres del final de la carrera. Así, la investigación no se establece como una necesidad u obligación dentro de la preparación profesional de los futuros profesores. De acuerdo con Perines y Campaña (2019) el lugar que la investigación ocupa en el currículo depende, generalmente, de las decisiones políticas de cada institución (Harris y Sass, 2011) y de los contextos propios de cada una de ellas. La incorporación de la investigación en las programaciones curriculares exige que las instituciones instauren e incluyan, de manera explícita, la investigación educa- 
tiva entre sus lineamientos formativos (Burn, 2007; Hagger y McIntyre, 2000; Lampert, 2009).

Desde la perspectiva de Anwaruddin y Pervin (2015), el compromiso de los docentes con la investigación educativa depende, entre otras cosas, de que hayan sido "alfabetizados" en ello, es decir, de que reciban educación sobre investigación cuando están estudiando pedagogía. Precisamente la formación en investigación (o la no-formación) es uno de los elementos criticados por los profesores en ejercicio en algunos estudios (p.ej., Murillo, Perines y Lomba, 2017; Perines y Murillo 2017a, Perines y Murillo, 2017b). Muchos docentes tienen una mirada suspicaz sobre la preparación en investigación que recibieron y la indican como una de las causas del rechazo o indiferencia hacia las evidencias científicas o hacia los artículos de investigación, cuando ya ejercen la pedagogía. En otros casos, los profesores en ejercicio tienen problemas para construir un marco teórico, ya que muchos de ellos lo realizan a través del sentido común, y no a partir de las evidencias de otros autores (Tovar-Gálvez, 2018).

Una forma concreta de "alfabetizar" a los futuros profesores es a través de la vinculación de los estudiantes de pedagogía a proyectos de investigación (Parra, 2004). Un ejemplo preciso de ello, puede ser, trabajar con "semilleros de investigación", entendidos como grupos disciplinares o interdisciplinares autónomos, donde estudiantes universitarios desarrollan estudios junto a docentes e investigadores, con el propósito de iniciar un camino formativo dentro del campo de la investigación (Hernández-Pino, 2005). Otra forma de sensibilizarlos con el tema es darle oportunidades concretas de observar problemas educativos provenientes de la realidad educativa, para que desde allí, puedan pensar en soluciones a dichas problemáticas.

En esta misma línea, Mansilla y Garrido (2019) afirman que la formación inicial de profesores debe avanzar hacia una didáctica de la metodología de la investigación escolar, con énfasis en la escuela y en sus necesidades. De acuerdo con estos autores, es importante que los programas de profesorado incluyan a la investigación dentro del currículum de manera transversal en el itinerario formativo, por medio de procesos reflexivos que surjan desde situaciones educativas reales.

Ahora bien, los procesos de alfabetización en investigación enfrentan ciertas dificultades; desde la perspectiva de Aldana de Becerra (2012), un ejemplo de estas dificultades es que la formación en investigación actual es lineal y fragmentada. Esta no motiva a los estudiantes a producir y reflexionar sobre el conocimiento, sino que se orienta a preparar al futuro profesor para que investigue "realmente" en estudios de posgrado y no en el grado. De acuerdo con este autor, la enseńanza de la investigación en los currículos antiguos es más informativa que formativa; muchos de los programas de pregrado incluyen a la investigación como una "receta" y "no como algo susceptible de ser comprendido, mejorado o completado" (p. 6).

En esta revisión teórica se mencionarán aquellos estudios que abordan la formación investigativa, pero desde las valoraciones de los agentes educativos involucrados.

Siguiendo un orden cronológico para describirlas encontramos que, en el año 2002, Ruíz y Torres desarrollan una investigación sobre la actitud de los estudiantes hacia el aprendizaje de la investigación en universidades venezolanas. Los hallazgos muestran que el $68 \%$ de los estudiantes tenían una actitud entre neutra y desfavorable hacia el aprendizaje de la investigación. Por otro lado, solo un 32 \% mostró una actitud entre favorable y muy favorable, y este grupo correspondió al área de ciencias básicas y de la salud. Dos años después, Torres (2004) realizó una investigación en la que comparó estrategias de enseñanza: receptivas activas. Los resultados muestran que cuando los profesores universitarios utilizan estrategias prácticas que enfatizan la enseńanza de las ciencias, los estudiantes logran mejores aprendizajes en comparación con la enseñanza tradicional.

En el año 2012, van der Linden et al. realizan un estudio centrado en la actitud de los estudiantes hacia un curso introductorio de investigación. Este mostró que los estudiantes consideran la investigación educativa más importante que la posible utilización práctica de una investigación en el futuro. Por otra parte, señalan que el aspecto menos útil del curso fue la falta 
de conexión con el programa de estudios general de la carrera.

Si bien los autores Larenas et al. (2015) no centran su estudio específicamente en futuros docentes, sí obtienen hallazgos relevantes sobre su formación en investigación. Los autores realizan un estudio de enfoque mixto que pretende analizar los temas más relevantes de la formación de profesores en Chile. Respecto a la formación inicial docente, el $65 \%$ de los docentes universitarios afirma enseñar herramientas investigativas a sus estudiantes, mientras que un $28 \%$ señala que forma a futuros investigadores.

En un trabajo más actual, Haberfellner y Fenzl (2017) obtuvieron resultados más optimistas al entrevistar a estudiantes de pedagogía sobre la importancia de la investigación educativa y sobre el impacto y utilidad de la investigación en la práctica educativa. Muchos de los entrevistados creen que obtener conocimiento sobre la investigación educativa les ayuda a prepararse para el trabajo científico, lo cual consideran vital para su formación. Asimismo, gran parte de los participantes está convencido de que la investigación educativa y la evidencia de la investigación aumenta su conocimiento pedagógico.

Desde una perspectiva centrada en los procesos didácticos que se dan en las pedagogías nace el concepto de Aprendizaje Basado en la Investigación (ABI). En el contexto latinoamericano, el Tecnológico de Monterrey en su sitio de Investigación e Innovación Educativa, lo define como "la aplicación de estrategias de enseñanza y aprendizaje que tienen como propósito conectar la investigación con la enseñanza, las cuales permiten la incorporación parcial o total del estudiante en una investigación basada en métodos científicos, bajo la supervisión del profesor" (2010, p. 208).

En esta línea, Peñaherrera, Chiluiza y Ortiz (2014) realizan una propuesta para incluir el Aprendizaje Basado en la Investigación (ABI) en el diseño académico de un programa de estudio. Los autores buscan que, al finalizar el programa, los estudiantes posean habilidades para investigar como parte del propio proceso y no el sentido tradicional de la inclusión de un curso aislado de métodos de investigación. La propuesta se basa en llevar a cabo 16 pasos, en los que se incluyen, por ejemplo, definir y agrupar temas de investigación a desarrollar en los cursos, buscar líderes de trabajo, entre otros. Los autores concluyen que el $\mathrm{ABI}$ es una estrategia importante de aplicar en la práctica pedagógica y destacan que su aplicación desarrolla habilidades de investigación como un proceso vivencial y no solo teórico.

Ahora bien, es importante también analizar qué competencias de investigación son relevantes en el contexto de la formación de profesorado. Diversos autores presentan una cierta coincidencia en la identificación de estas competencias (p. ej., Pirela y Prieto, 2006; Gray, 2007; Meerah y Halim, 2012; Valdés, Vera y Carlos, 2012). Algunos de estos elementos son, por ejemplo: identificar, plantear y formular problemas, objetivos o hipótesis, manejar fuentes de información, habilidades de resolución de problemas científicos y prácticos, habilidades de comunicación y escritura de los resultados, actitudes personales y ética profesional, roles del investigador mediante la contribución al desarrollo del conocimiento y el diseño de estudios y la publicación, entre otras (Rubio, et al. 2016, p. 4).

$\mathrm{Si}$ algo se reitera en esta revisión teórica es que se necesitan nuevas formas de interacción donde los futuros profesores puedan vincularse con la investigación (Zeichner, 1995, 2010). Lograrlo exige, entre otras cosas, un incremento de las oportunidades de aprendizaje en investigación, como también de mejores instancias de interacción con sus formadores (Korthagen, 2010).

Los futuros profesores deberían ser capaces de reflexionar críticamente y aplicar literatura de investigación para su contexto específico. Si los docentes de pregrado reconocen el valor que tienen las evidencias para su práctica, se puede generar una incidencia en la forma en que abordan los conocimientos que produce la investigación (Eccles y Wigfield, 2002; Vetter y Ingrisani, 2013).

La generación y el uso del conocimiento son principios fundamentales para otorgar un sentido más profesional y reflexivo a la labor docente. No obstante, la 
capacidad de reflexión no parece ser un aspecto esencial en la identidad del profesor, al menos, así lo refleja el último informe de la evaluación docente chilena, donde la "reflexión a partir de los resultados de la evaluación" es la dimensión donde hay más bajo desempeño (Ministerio de Educación de Chile, 2016). Tampoco hay suficiente evidencia de que los procesos formativos del profesor se hayan encargado de ello, al menos en forma intencionada y planificada (Catalán, 2011, 2017; Perrenoud, 2007). Asimismo, "la formación inicial docente en Chile enfrenta el desafío de buscar un sistema de atracción a la profesión docente, que permita que los futuros profesores se conviertan en agentes reflexivos y no sólo en transmisores mecánicos del currículum" (Larenas et al., 2015, p. 4). La reflexividad del profesor favorecerá la calidad de la docencia, ya que les permitirá replantearse sus propias creencias y tomar decisiones con autonomía (Scherping, 2010).

Finalmente, resulta pertinente mencionar los tres ámbitos en que, según Forner (2000, p. 41), debe mejorar la relación entre la investigación y la formación del profesorado:

1. La investigación en la formación: se refiere al incremento de la presentación y análisis de estudios e investigaciones como recurso en las materias curriculares.

2. La investigación para la formación: se refiere al uso de la investigación sobre contenidos curriculares por parte de los propios estudiantes, como una opción de mejora de los procesos de enseñanza-aprendizaje y como alternativa a las rutinas tradicionales de los docentes.

3. La formación para la investigación: se refiere a la capacitación de los estudiantes en materias de investigación para que puedan colaborar y desarrollar proyectos de investigación vinculados a su práctica profesional.

\section{Metodología}

El objetivo de este estudio es determinar la presencia de la investigación educativa en los planes y programas de formación y en el perfil de egreso de las carreras de pedagogía de la Universidad de La Serena (Chile). Como se ha mencionado, este propósito también es el primer objetivo establecido en el proyecto Fondecyt $\mathrm{N}^{\circ} 11180227$. De forma concreta, se realizó un estudio cualitativo, utilizando la técnica del análisis documental. Asimismo, se realizó un análisis de los once planes y programas de estudio antiguos (vigentes hasta 2018) y de los renovados para el año 2019.

El análisis documental busca describir y representar los documentos de forma sistemática para facilitar su recuperación (Dulzaides y Molina, 2004). La finalidad de este tipo de análisis es simplificar el contenido de los documentos y representarlos de una forma diferente a la original, tomando solo sus elementos esenciales o referenciales (Peña Vera y Pirela Morillo, 2007).

La complejidad de realizar un análisis documental es que este adolece de una metodología de trabajo definida. Es por ello que el análisis del discurso se constituye como un marco metodológico para el análisis documental, entendiendo "que los documentos son discursos expresados a través de textos, $y$, por consiguiente, su estructura discursiva puede ser abordada a partir de los lineamientos y procesos aplicados en el análisis del discurso" (Peña Vera y Pirela Morillo, 2007, p. 9). Por estos motivos, se han utilizado como marco referencial las macrorreglas propuestas por van Dijk (1980), las que orientaron el análisis del discurso presente en cada uno de los documentos institucionales.

De acuerdo con Peña Vera y Pirela Morillo (2007, p. 12), las macrorreglas de van Dijk permiten "reconstruir la capacidad lingüística del individuo, enlazando significados y convirtiéndolos en totalidades significativas más grandes; su característica principal es su naturaleza organizadora, por cuanto le imprimen unidad a las proposiciones, las cuales son la representación abstracta de la información".

Estas macrorreglas son:

1. Omitir: que consiste en suprimir la información que no sea relevante.

2. Seleccionar: consiste en discriminar elementos del texto, lo cual requiere también omitir alguna información. 
3. Generalizar: consiste en generar una proposición generalizante o abstracción que abarque otros elementos.

4. Construir o integrar: consiste en generar una proposición o concepto más general que aborde la totalidad del discurso.

A través de este razonamiento se indagó en los programas de estudio y en el perfil de egreso de las carreras de pedagogía, tanto de los programas antiguos como de los que están en proceso de renovación para el año 2019. En ambos casos se identificaron las asignaturas que trabajan explícitamente con elementos propios de la investigación educativa, utilizando principalmente la macrorregla de la selección. También se indagó en la descripción detallada de cada una de estas asignaturas: descripción, objetivos, contenidos, evaluación, bibliografía en el caso de los planes antiguos; y descripción, estándares, resultados de aprendizaje, contenidos, estrategias metodológicas, evaluación y bibliografía, en el caso de los programas renovados. En este caso, se utilizaron en distinta medida, las cuatro macrorreglas: omitir, seleccionar, generalizar y construir. La macrorregla omitir fue menos utilizada ya que se intentó revisar la totalidad del escrito. Se utilizó la macrorregla de seleccionar al momento de revisar la descripción de la asignatura y los contenidos considerando que estos dos ítems proporcionan información relevante respecto al fundamento que tiene la asignatura. La generalización se utilizó fundamentalmente cuando se conoció el contenido de todas las asignaturas analizadas y se determinó una mirada común en todas ellas. La macrorregla de construir se dio cuando se redactaron los resultados del estudio.

Posteriormente se analizó la presencia de la investigación educativa en los estándares y perfiles de egreso de cada una de las carreras. En el caso de los estándares, llamados competencias en otros contextos, se utilizó principalmente la macrorregla de seleccionar, al tomar solo aquellos estándares relacionados de una $\mathrm{u}$ otra forma con la investigación educativa. En el caso de los perfiles de egreso se utilizaron las macrorre- glas de seleccionar, generalizar y construir, ya que luego de la lectura general se procedió a seleccionar aquellos apartados u oraciones vinculados directamente con la investigación, para luego extraer generalidades y construir la teoría.

\subsection{Fuentes de información y contexto}

\section{Las carreras de pedagogías de la Universidad} de La Serena

Realizar este estudio en la Universidad de la Serena (ULS) se fundamenta en dos motivos principales. El primero de ellos es que la ULS es la Universidad Estatal del Norte de Chile que cuenta con una mayor cantidad de carreras de pedagogía, las que en total son 11, que se mencionan a continuación:

1. Pedagogía en Educación General Básica

2. Pedagogía en Educación Parvularia

3. Pedagogía en Educación Diferencial

4. Pedagogía en Matemáticas y Física

5. Pedagogía en Matemáticas y Computación

6. Pedagogía en Química y Ciencias Naturales

7. Pedagogía en Biología y Ciencias Naturales

8. Pedagogía en Historia y Geografía

9. Pedagogía en Inglés

10. Pedagogía en Educación Musical

11. Pedagogía en Castellano y Filosofía

Con esta oferta académica, la Universidad de La Serena entrega a la sociedad la mayoría de los docentes en ejercicio de la Región de Coquimbo, e incluso, también de la Región de Atacama. Este escenario convierte a esta universidad en un fructífero campo de estudio para conocer la realidad de sus programas de estudio.

Por otro lado, las carreras de pedagogía de la Universidad de La Serena están viviendo actualmente un importante proceso de cambios, que se enmarca en el Proyecto de Mejora Institucional FIP1501. Este proyecto, financiado por Programa de Mejoramiento de la Calidad y Equidad en la Educación (MECESUP) 
se inició el año 2016. Uno de sus objetivos específicos es "resideñar el currículo de las pedagogías de acuerdo con la concepción institucional de un profesor de calidad, en coherencia con su modelo educativo que se centra en el aprendizaje, y a los lineamientos del MINEDUC expresados" (p. 12).

De acuerdo con este propósito se hizo un rediseño de los programas entre los años 2017 y 2018, proceso en el que se buscó establecer criterios comunes mínimos para las once carreras, especialmente en los estándares generales y pedagógicos, así como también en la definición de la licenciatura en educación. Los programas rediseñados iniciaron su fase de implementación en el curso académico del año 2019 (en el mes de abril específicamente), con los estudiantes que ingresaron a primer año.

Este escenario de cambios es propicio para analizar la presencia e importancia de la investigación educativa en la transición de los programas antiguos y renovados y ver sus alcances en términos curriculares. Es importante mencionar que los estudiantes de segundo, tercero, cuarto y quinto continúan con los planes y programas antiguos, los que quedarán obsoletos una vez que egrese la generación que inició sus estudios en abril del 2019.

\section{Resultados}

Los resultados del análisis se organizan de acuerdo con la presencia de la investigación educativa en: los perfiles de egreso antiguos, perfiles de egreso renovados, programas de estudio antiguos y programas de estudio renovados.

\subsection{Presencia de la investigación educativa en los perfiles de egreso antiguos}

En los programas de estudio antiguos no existe un apartado que indique explícitamente el concepto de "perfil de egreso".

En los documentos oficiales se hace una presentación de la carrera (véase figura 1) donde la "descripción" y las "aptitudes deseables" son elementos que podrían aludir a un posible perfil de egreso.

Figura 1.

Presentación de la carrera de pedagogía en educación parvularia

\section{CARRERA: PEDAGOGIA EN EDUCACIÓN PARVULARIA}

\begin{tabular}{|c|c|}
\hline \multicolumn{2}{|c|}{$\begin{array}{l}\text { Titulo Profesional: Educador (a) de Párvulos } \\
\text { Grado Académico: Licenciado en Educación } \\
\text { Duración: } 8 \text { semestres }\end{array}$} \\
\hline $\begin{array}{l}\text { Requisitos de Ingreso: } \\
\text { * Enseñanza Media: } \\
\text { * Ranking: } \\
\text { * PSU Lenguaje y Comunicación: } \\
\text { * PSU Matemáticas: } \\
\text { * PSU Historia y Ciencias Sociales: }\end{array}$ & $\begin{array}{l}30 \% \\
20 \% \\
20 \% \\
15 \% \\
15 \%\end{array}$ \\
\hline \multicolumn{2}{|c|}{$\begin{array}{l}\text { Descripción: Profesional de la educación, con } \\
\text { profundo enfoque humanista, competente } \\
\text { para responder al desafío de educar en nivel de } \\
\text { Educación Parvularia, con cientificidad, afecto, } \\
\text { creatividad y dinamismo, a fin de liderar y } \\
\text { contribuir al desarrollo de las potencialidades de } \\
\text { niñas y niños en una concepción de respeto como } \\
\text { sujeto-persona que se construye junto a otros/as. }\end{array}$} \\
\hline
\end{tabular}

La carrera de Pedagogía en Educación Parvularia tiene un sello pedagógico-comunitario.

Campo Ocupacional: Jardines infantiles, como también en aquellos dependientes de organismos de la administración pública y privada (JUNJI, INTEGRA, MINEDUC), hospitales e instituciones de Educación Superior; docencia e investigación.

Aptitudes Deseables:

Vocación de servicio social a través de la enseñanza. Compromiso reflexivo y de constante búsqueda de soluciones a situaciones de la realidad cambiante. Apertura al trabajo interdisciplinario e interinstitucional.

Respeto y valoración hacia la persona del párvulo. Valoración de su rol como mediador de aprendizajes.

\begin{abstract}
Vacantes: 40
Régimen de Estudios: Semestral Continuidad de Estudios: Magíster en Educación, Doctorado, otros.

Informaciones: Campus Isabel Bongard, Escuela de Educación y Coordinación de Carrera, Amunátegui 851, La Serena.

Teléfonos: 51- 2204341 Escuela de Educación. 51-2204155/51-2204147

Coordinación de Carrera.
\end{abstract}

Carrera Acreditada, 6 años, Diciembre 2015 Diciembre 2021. Modalidad Presencial, Sede La Serena, Jornada Diurna (Agencia Acreditadora: Acreditacción).

Nota: Malla Curricular Pedagogía en Educación Parvularia, Universidad de La Serena, año 2018. 
Como vemos en el ítem "descripción”, se habla de un enfoque humanista, del afecto, la creatividad, el dinamismo, pero no se alude a la investigación educativa en ningún aspecto. Lo mismo sucede en las "aptitudes deseables" donde, por ejemplo, en el caso de Pedagogía en Educación Parvularia, se menciona, entre otras cosas, la vocación de servicio social, el compromiso reflexivo y el respeto hacia los niños, pero nuevamente la investigación educativa está totalmente ausente. Ahora bien, si revisamos lo que sucede en las "aptitudes deseables" de las otras diez carreras de pedagogías, se observa que en algunas de ellas se mencionan elementos de la investigación educativa, pero con poca precisión, por ejemplo:

- Pedagogía en Biología y Ciencias Naturales: "facilidad para los estudios científicos".

- Pedagogía en Educación Musical: "interés por la investigación".

- Pedagogía en Historia y Geografía: “espíritu indagador".

- Pedagogía en Matemáticas y Física: "facilidad para el estudio en ciencias experimentales y teóricas".

Con estos elementos ha quedado en evidencia que no existe una presencia generalizada ni tampoco sistematizada de la investigación educativa en los perfiles de egreso. Cabe mencionar que los planes y programas renovados, al comenzar su implementación en el primer semestre del año 2019, afectarán solo a los estudiantes de primer año. Los alumnos y alumnas de segundo a quinto año seguirán cursando las asignaturas del programa actual con su respectivo perfil de egreso.

\subsection{Presencia de la investigación en los perfiles de egreso renovados}

El decreto exento de cada uno de los planes renovados comienza con una descripción del perfil del egreso en coherencia con la especialidad de cada carrera, por lo que este elemento varía en cada una de ellas. Por ejemplo, el perfil de egreso de Pedagogía en Historia y Geografía es el siguiente:

El profesor/a de Estado en Historia y Geografía con su grado de Licenciado/a en Educación, egresado/a de la Universidad de la Serena, es un profesional de la educación que se caracteriza por comprender la construcción del conocimiento en el ámbito disciplinario y didáctico del contenido.

Reconoce su rol docente, indagando y reflexionando sobre su práctica pedagógica en diversos contextos de la cultura escolar. Como pedagogo/a de Historia y Geografía, está capacitado/a para estimular la inserción de las nuevas generaciones en el mundo actual, propiciando en sus estudiantes el desarrollo del pensamiento histórico y geográfico, contribuyendo a su formación ciudadana y desarrollo integral (Decreto exento $\mathrm{N}^{\circ}$ 269/2018, p. 1).

Luego, vemos que la primera novedad de los programas renovados es la incorporación de estándares genéricos (habilidades y capacidades generales que deben desarrollar los estudiantes en el área de formación general comunes a las once pedagogías), estándares pedagógicos (habilidades y capacidades vinculadas directamente con la licenciatura en educación comunes a las once pedagogías) y estándares disciplinares (referidos a las habilidades y capacidades que varían de acuerdo con cada especialidad).

Dentro de los estándares genéricos (que son un total de 10) hay uno de ellos que dice: "capacidad de abstracción, análisis y sintesis". Si bien en dicho estándar no se menciona explícitamente la expresión "investigación educativa”, sí se alude a las habilidades propias de los procesos investigativos, por lo que, para efectos de este estudio, sí se considera como un elemento relacionado con la investigación educativa. En cuanto a los estándares pedagógicos (que son un total de 7) uno de ellos dice: "capacidad investigativa y de reflexión critica sobre su práctica pedagógica, los procesos educativos y las relaciones en la comunidad". En este caso, hay una clara alusión a la investigación educativa, que se vincula con elementos de reflexión sobre la práctica pedagógica.

Otro elemento que se incorpora en los perfiles de egreso renovados es el perfil del licenciado en educación, que va en coherencia a los estándares pedagógicos y que es común para las once carreras. Este perfil es el siguiente: 
Graduado/a universitario/a que posee conocimientos de la estructura conceptual y epistemológica de las ciencias de la educación, de los fenómenos y situaciones educativas, y conocimientos básicos de investigación educacional para analizar los problemas educativos y generar conocimientos profesionales asociados al mejoramiento de su propio desempeńo, de la unidad educativa de pertenencia y de los contextos próximos (Decreto exento $\mathrm{N}^{\circ}$ 269/2018)

Este perfil del licenciado en educación alude directamente a la investigación educativa cuando dice: "conocimientos básicos de investigación educacional para analizar los problemas educativos y generar conocimientos profesionales asociados al mejoramiento de su propio desempeńo, de la unidad educativa de perte- nencia y de los contextos próximos" (Decreto exento $N^{\circ}$ 269/2018). Esta inclusión deja en evidencia que la mirada hacia el tema, tanto en dicho perfil como en la presencia de estándares, es bastante diferente a lo observado en los perfiles antiguos.

\subsection{Presencia de la investigación educativa en los planes antiguos}

En los planes antiguos se observan las asignaturas propias de la disciplina y las relativas a la formación pedagógica, las que son comunes para las once carreras de pedagogía. Al observar la malla curricular en su totalidad, se evidencia la presencia de cuatro asignaturas relacionadas con la investigación educativa, las que se muestran en la tabla 1.

\section{Tabla 1}

Asignaturas relacionadas con la investigación educativa en los programas antiguos (vigentes hasta 2018)

\begin{tabular}{|c|c|c|}
\hline $\begin{array}{l}\text { Nombre } \\
\text { asignatura }\end{array}$ & Semestre en que se imparte & Breve descripción \\
\hline 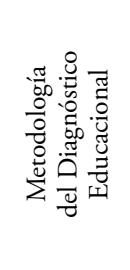 & $\begin{array}{l}\text {-1er semestre: Pedagogía en Educación Diferencial, Pedagogía en } \\
\text { Educación General Básica, Pedagogía en Educación Musical. } \\
\text {-2do semestre: Pedagogía en Castellano y Filosofía, Pedagogía } \\
\text { en Educación Parvularia, Pedagogía en Inglés, Pedagogía en } \\
\text { Matemáticas y Computación, Pedagogía en Matemáticas y } \\
\text { Física, Pedagogía en Química y Ciencias Naturales, Pedagogía en } \\
\text { Biología y Ciencias Naturales. }\end{array}$ & $\begin{array}{l}\text { Esta asignatura posee un carácter teórico y práctico y está orientada a lograr } \\
\text { un conocimiento riguroso del diagnóstico educacional, entendido éste como } \\
\text { un proceso de investigación aplicada, dirigido al conocimiento de contextos, } \\
\text { interacciones y problemas educativos; y cuya finalidad es buscar, fundamentar } \\
\text { e implementar soluciones a los mismos. }\end{array}$ \\
\hline 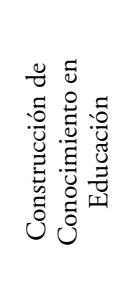 & $\begin{array}{l}\text {-2do semestre: Pedagogía en Educación Parvularia. } \\
\text {-3er semestre: Pedagogía en Educación Diferencial, Pedagogía en } \\
\text { Educación General Básica, Pedagogía en Castellano y Filosofía, } \\
\text { Pedagogía en Inglés, Pedagogía en Biología y Ciencias Naturales. } \\
\text {-4to semestre: Pedagogía en Historia y Geografía, Pedagogía en } \\
\text { Matemáticas y Computación, Pedagogía en Matemáticas y Física. } \\
\text {-5to semestre: Pedagogía en Química y Ciencias Naturales }\end{array}$ & $\begin{array}{l}\text { La asignatura posee un carácter eminentemente teórico, la cual, } \\
\text { contempla la revisión crítica de las bases epistemológicas que fundamentan la } \\
\text { construcción del conocimiento en el ámbito de la educación. Con tal objeto se } \\
\text { analizan las discusiones acerca de la estructura del conocimiento, los principios } \\
\text { que la sostienen y la relación con los principios educativos. }\end{array}$ \\
\hline 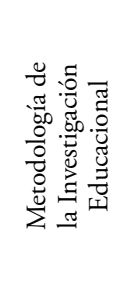 & $\begin{array}{l}\text {-Gto semestre: Castellano y Filosofía, Educación Parvularia, } \\
\text { Inglés. } \\
\text {-7mo semestre: Educación Diferencial, Educación General } \\
\text { Básica, Educación Musical, Historia y Geografía, matemáticas } \\
\text { y Computación, Matemáticas y Física, Química y Ciencias } \\
\text { Naturales, Biología y Ciencias Naturales. }\end{array}$ & $\begin{array}{l}\text { Esta asignatura de enfoque teórico-práctica se vincula al campo de la } \\
\text { investigación educacional y a sus alcances de carácter científico, educacional } \\
\text { y social. El curso se constituye en una instancia de acercamiento, desde } \\
\text { una perspectiva teóricamente sustentada, el análisis y discusión crítica de } \\
\text { problemáticas actuales ligadas al ámbito de la educación. De manera paralela, } \\
\text { se busca desarrollar en el estudiante las competencias necesarias para la } \\
\text { elaboración colectiva de un proyecto de investigación, por medio del cual se } \\
\text { evidencie la apropiación de los protocolos base de toda investigación científica. }\end{array}$ \\
\hline 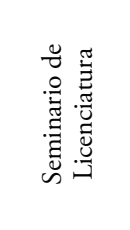 & $\begin{array}{l}\text {-7mo semestre: Castellano y Filosofía, Educación Parvularia, } \\
\text { Inglés. } \\
\text {-8vo semestre: Educación Diferencial, Educación General } \\
\text { Básica, Educación Musical, Historia y Geografía, Matemáticas } \\
\text { y Computación, Matemáticas y Física, Química y Ciencias } \\
\text { Naturales, Biología y Ciencias Naturales. }\end{array}$ & $\begin{array}{l}\text { Es una asignatura de carácter teórico-práctico que profundiza en aspectos } \\
\text { relacionados con la evaluación de una propuesta de investigación, el análisis } \\
\text { de las condiciones para su puesta en práctica, la aplicación de los instrumentos } \\
\text { y/o técnicas seleccionadas (trabajo de campo), la interpretación, en un primer } \\
\text { nivel de análisis, de los datos obtenidos y el desarrollo de las conclusiones del } \\
\text { estudio. }\end{array}$ \\
\hline
\end{tabular}

Nota: elaboración propia a partir de los programas de pedagogía de la Universidad de La Serena vigentes hasta 2018. 
Llama la atención que el semestre en que se imparte cada asignatura varía en algunas carreras, lo que no presenta una lógica pedagógica definida. Al parecer esto obedece netamente a decisiones de cada carrera y a la distribución definida por cada una de ellas.

En la descripción de las cuatro asignaturas vemos que Metodología del Diagnóstico Educacional tiene un enfoque teórico y práctico, mientras que Construcción del Conocimiento en Educación es fundamentalmente teórica. Por su parte, Metodología del Diagnóstico Educacional tiene una orientación de teoría y práctica, al igual que Seminario de Licenciatura.

Ahora bien, si se presta una mayor atención a la descripción de cada una de ellas, se evidencia que
Seminario de Licenciatura es la que tiene un enfoque más "concreto" o de poner en práctica los conocimientos adquiridos. Esto tiene cierta coherencia con el nivel alcanzado por los estudiantes al estar en séptimo u octavo semestre, por lo que se infiere que en los programas antiguos privilegia un tratamiento más bien teórico sobre la investigación educativa, para en el último semestre darle una orientación más cercana al contexto educativo real.

Para graficar la distribución de estas cuatro asignaturas, en el transcurso de la carrera se incorpora la figura 2, que expone la malla curricular antigua de Pedagogía en Educación General Básica:

Figura 2

Asignaturas de la carrera

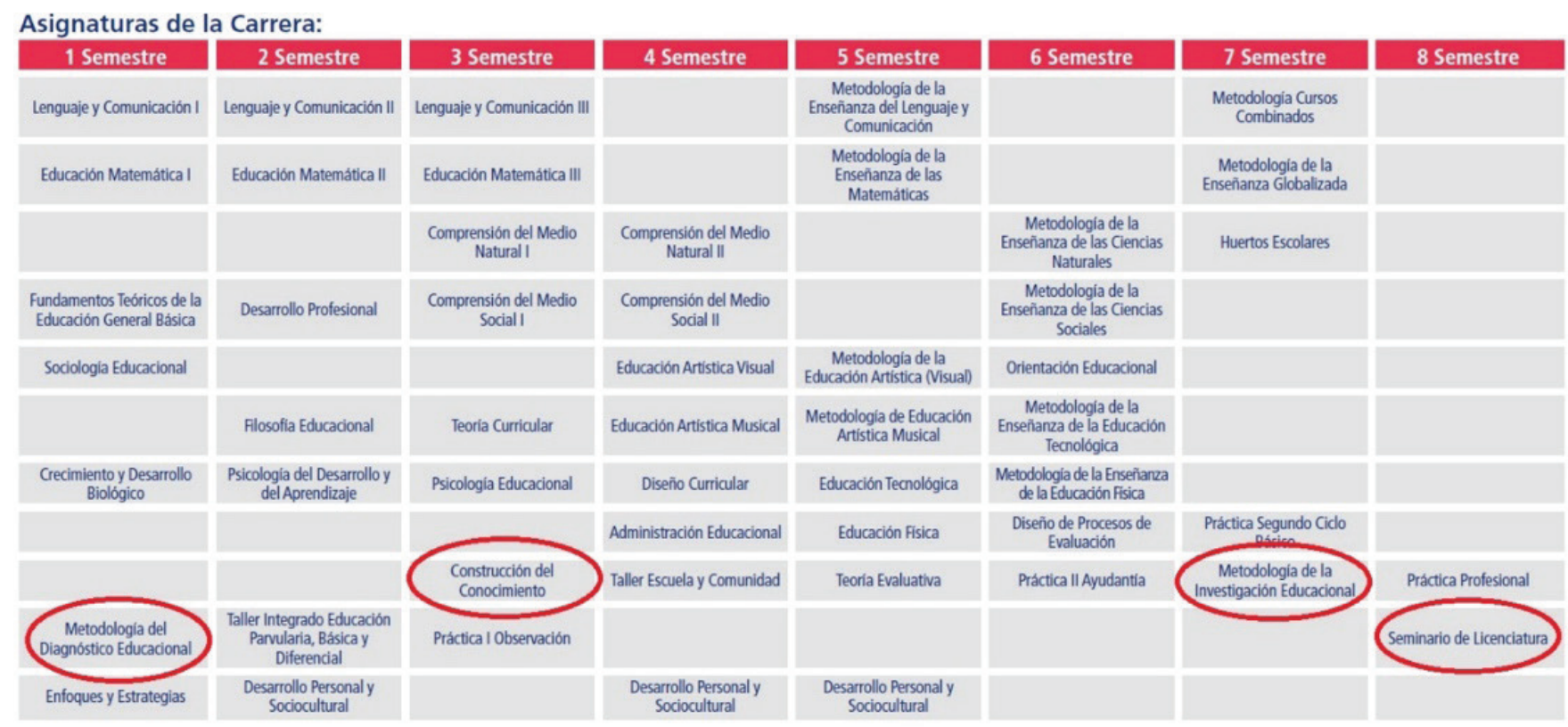

Nota: Malla Curricular Pedagogía en Educación General Básica, Universidad de La Serena, año 2018.

Lo expuesto en la figura deja de manifiesto que no existe una vinculación o una interrelación entre cada una de las asignaturas, con la excepción de Metodología de la Investigación Educacional, que es pre-requisito para el Seminario de Licenciatura. Las cuatro asignaturas vinculadas a la investigación se dan en semestres discontinuos y, como ya se dijo, no existe uniformidad respecto al semestre en que la asignatura se da en cada carrera. 


\subsection{Presencia de la investigación educativa en los programas renovados}

En los planes renovados y en proceso de implementación desde el año 2019, el primer cambio que queda a la vista es que todas las asignaturas que desarrollan elementos de la investigación educativa tienen un orden secuencial, y cada una es pre-requisito de la siguiente. Asimismo, se cambian parcialmente las asignaturas del plan antiguo y se agrega una más, teniendo un total de cinco asignaturas vinculadas con la investigación, las que se mencionan en la tabla 2 .

Tabla 2

Asignaturas relacionadas con la investigación educativa en los programas renovados (vigentes desde 2019)

\begin{tabular}{|c|c|}
\hline Nombre asignatura & $\begin{array}{c}\text { Año en que se } \\
\text { imparte }\end{array}$ \\
\hline Teorías de la educación & 4to semestre \\
\hline $\begin{array}{c}\text { Epistemología y Paradigmas de la Investigación } \\
\text { Educativa }\end{array}$ & 5to semestre \\
\hline Análisis de Proyectos de Investigación Educativa & 6to semestre \\
\hline Metodología de la Investigación Educativa & 7mo semestre \\
\hline Seminario de Licenciatura en Educación & 8vo semestre \\
\hline
\end{tabular}

Nota: elaboración propia a partir del Decreto Exento N²94/2018.

A la fecha, cada carrera y/o cada departamento continúa elaborando los programas, por lo que aquellos programas mencionados en la figura 2, no se encuentran a disposición para ser analizados. Sin embargo, es posible establecer algunos alcances a partir de los nombres de las asignaturas. Las asignaturas Teorías de la Educación y Epistemología y Paradigmas de la Investigación Educativa tienen un enfoque más bien teórico y de adquisición de conocimientos iniciales sobre la investigación educativa. Por otra parte, las asignaturas Análisis de Proyectos de Investigación Educativa, Metodología de la Investigación Educativa y Seminario de Licenciatura, eventualmente tienen un enfoque más vinculado a la práctica pedagógica y al análisis de ella a partir de la investigación.

Para graficar la distribución de estas asignaturas en el transcurso de la carrera se incorpora la figura 3, que expone la malla curricular renovada de Pedagogía en Biología y Ciencias Naturales:

\section{Figura 3}

Malla curricular renovada de Pedagogía en Biología y Ciencias Naturales
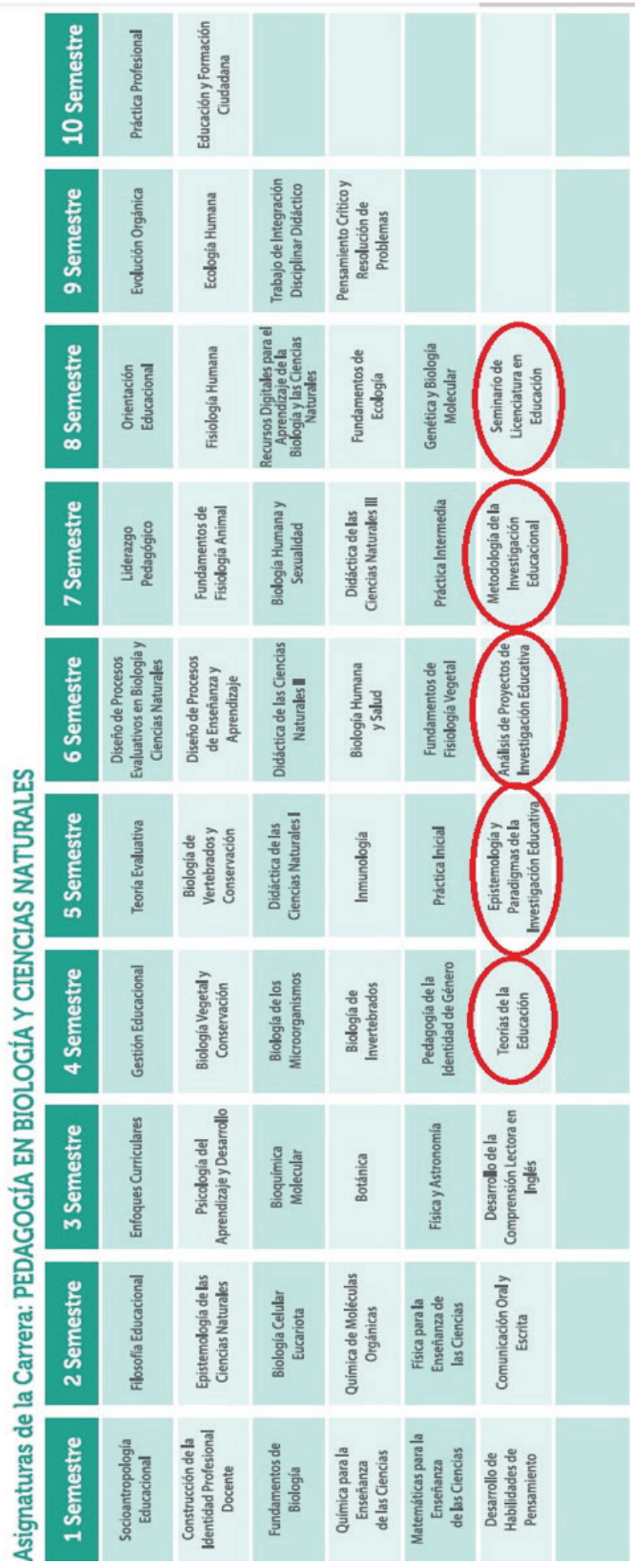

Nota: elaboración propia a partir del Decreto Exento 300/2018. 
En la figura expuesta queda en evidencia que las asignaturas vinculadas a la investigación educativa siguen una secuencia y que cada una tiene como pre-requisito a anterior. También es importante mencionar que estas asignaturas tienen la misma lógica en las once pedagogías. Ambos factores muestran diferencias contundentes entre los planes antiguos y renovados, especialmente en la distribución de las asignaturas.

\section{Discusión y conclusiones}

Antes de iniciar la discusión y las conclusiones es importante recordar que el principal objetivo de este trabajo es determinar la presencia de la investigación educativa en los planes y programas de las carreras de pedagogía de la Universidad de La Serena (Chile), lo que incluye los programas antiguos y los renovados. El primer elemento que queda en evidencia desde un punto de vista comparativo es que existen claras diferencias entre los programas y perfiles de los planes antiguos y los renovados. Mirando estos hallazgos a la luz de los estudios centrados en este tema, vemos que, en coherencia a lo mencionado por Aldana de Becerra (2012), la formación en investigación en los programas antiguos es más bien fragmentada. Por otra parte, en los programas renovados existe una mejor intención de que la investigación formativa puede integrarse en el plan de estudios de modo progresivo en profundidad y extensión, en coherencia a lo indicado por Parra (2004).

Respecto a las competencias de investigación destacadas por algunos autores (p. ej., Pirela y Prieto, 2006; Gray, 2007; Meerah y Halim, 2012; Valdés et al., 2012), ya mencionadas en el marco teórico, ha quedado en evidencia que estas se intentan abordar tanto en los programas antiguos como en los renovados, específicamente en la asignatura "Metodología de la Investigación Educativa". Sin embargo, estos resultados permiten también cuestionar si una asignatura es suficiente para desarrollar todas las competen- cias mencionadas. Si bien en ambos planes se incluyen otras asignaturas relacionadas con la investigación, estas parecen tener un enfoque más bien teórico, por ejemplo: Construcción del Conocimiento en los planes antiguos y Teorías de la educación en los planes renovados. Surge una esperanza cuando vemos que en los planes renovados se agrega Análisis de Proyectos de Investigación en el sexto semestre, cátedra que debería tener un enfoque práctico y donde deberían trabajar las habilidades de investigación ya señaladas.

En este análisis ha quedado en evidencia que los planes y programas renovados expresan una mayor intencionalidad de incluir a la investigación educativa como un elemento central e importante dentro de la formación académica de un futuro profesor. Esto tiene cierta lógica en un contexto de renovación curricular, donde es inherente que se optimicen ciertos aspectos. Sin embargo, a partir de lo que señala la literatura vigente, queda la sensación de que no basta con la inclusión explícita de asignaturas, pues se requiere de más capacitaciones en investigación de los formadores y una real concientización sobre la importancia de la investigación educativa en el desarrollo profesional de los futuros docentes. Puede que se optimicen las asignaturas o se agreguen algunas, pero si no se orienta a los formadores para que se apropien de la investigación como recurso tangible y necesario, los cambios quedarán en el plano superficial.

Cabe recalcar que este es el primer estudio en Chile que analiza la presencia e importancia de la investigación educativa en los programas de formación docente, siendo todavía más pionero en el contexto de la Universidad de La Serena, universidad de la zona norte de Chile con la mayor oferta académica de pedagogías.

Analizar los programas de pedagogía es una deuda pendiente que tenemos los investigadores educativos. Mucho se ha dicho sobre las valoraciones de los estudiantes sobre la investigación educativa, pero poco sobre cómo se articulan los programas y sus asignaturas respecto a ella. 
En esta línea, las principales contribuciones de esta investigación son, por una parte, aportar datos a la literatura vigente sobre la formación en investigación de los futuros profesores. Por la otra, se pretende ayudar a las universidades y a la propia Universidad de La Serena a observar de manera analítica la forma en que se incluye la investigación educativa dentro de los programas de estudio, así como mirar con ojos críticos la calidad de los procesos de renovación. Los nuevos programas deben ir más allá de cambios "en el papel" y debe existir un trabajo intencionado para optimizar la formación profesional.

La principal limitación de este estudio es que se realiza solo en el contexto de una universidad. Tal vez podría ser de interés desarrollar un estudio más amplio con más planes y programas universitarios tanto en Chile como en el extranjero. Otro desafío, mirando hacia el futuro de esta temática, es profundizar en las valoraciones que los propios docentes universitarios o los estudiantes tienen sobre los programas de pedagogía o sobre las renovaciones curriculares de estos. Tal vez haga falta evaluar el impacto real de las asignaturas en la realidad cotidiana de un profesor o profesora. Una manera de lograrlo es realizar estudios longitudinales que analicen la calidad de los programas renovados, o también investigaciones que analicen las valoraciones de los futuros profesores sobre la formación en investigación recibida o sobre las intenciones de utilizar la investigación, una vez que ingresen a la vida laboral (p. ej. Griffioen, 2018).

Los resultados de este estudio son importantes para la comunidad educativa nacional e internacional, porque en ellos se refleja la necesidad de incluir a la investigación educativa en los programas más allá de un salto cuantitativo. Esta inclusión no solo se trata de contar con asignaturas que traten a la investigación, sino que se requiere de un cambio más profundo al interior de las carreras, facultades y universidades. Un cambio que valide a la investigación educativa como un elemento fundamental en la formación de un profesor bien preparado para el mundo laboral. Un profesional reflexivo, capacitado y actualizado y sobre todo consciente de que las evidencias científicas pueden ser un aporte para lograr una educación de mejor calidad.

\section{Referencias}

Aldana de Becerra, G. M. (2012). La formación investigativa: su pertinencia en el pregrado. Revista Virtual Universidad Católica del Norte, 1(35), 367-379.

Anwaruddin, S. M. y Pervin, N. (2015). English-language teachers' engagement with research: Findings from Bangladesh. Professional Development in Education, 41(1), 21-39. https://doi. org/10.1080/19415257.2013.861860

Burn, K. (2007). Professional knowledge and identity in a contested discipline: challenges for student teachers and teacher educators. Oxford Review of Education, 33(4), 445-467. https://doi.org/ 10.1080/03054980701450886

Cain, T. y Allan, D. (2017). The invisible impact of educational research. Oxford Review of Education, 43(6), 718-732. https://doi.org/10.1080/ 03054985.2017 .1316252

Catalán, J. (2011). Del pensamiento al conocimiento profesional del profesor. En J. Catalán. Psicología educacional: proponiendo rumbos, problemáticas y aportaciones (pp.187-215). La Serena: Editorial Universidad La Serena.

Catalán, J. (2017). Investigación y Docencia: una vinculación necesaria y posible. En F. Araya (Coord.) Desarrollo del Pensamiento Geográfico: Aportes para la vinculación entre Investigación Docencia (pp.17-32). La Serena: Editorial Universidad de La Serena.

Dulzaides, M. E. y Molina, A. M. (2004). Análisis documental y de información: dos componentes de un mismo proceso. Acimed, 12(2), 1-1. 
Eccles, J. S.yWigfield, A. (2002). Motivational beliefs, values, and goals. Annual review of psychology, 53, 109-132. Doi: https://doi.org/10.1146/ annurev.psych.53.100901.135153

Forner, A. (2000). Investigación educativa y formación del profesorado. Revista Interuniversitaria de Formación del Profesorado, (39), 33-50.

Goodyear, P. y Zenios, M. (2007). Discussion, collaborative knowledge work and epistemic fluency. British Journal of Educational Studies, 55(4), 351-368.

Gray, C. (2007). Research Competencies Framework. England: Faculty of General Dental Practice (UK). The Royal College of Surgeons of England.

Griffioen, D. M. (2018). The influence of undergraduate students' research attitudes on their intentions for research usage in their future professional practice. Innovations in Education and Teaching International, 56(2), 162-172. https://doi.org/1 $0.1080 / 14703297.2018 .1425152$

Guilbert, D., Lane, R. yVan Bergen, P. (2015). Understanding student engagement with research: a study of pre-service teachers' research perceptions, research experience, and motivation. Asia-Pacific Journal of Teacher Education, 44(2), 172-187. 10.1080/1359866X.2015.1070118

Haberfellner, C. y Fenzl, T. (2017). The utility value of research evidence for educational practice from the perspective of preservice student teachers in Austria-A qualitative exploratory study. Journal for Educational Research Online, 9(2), 69-87.

Hagger, H. y McIntyre, D. (2000). What can research tell us about teacher education? Oxford Review of Education, 26(3-4), 483-494. https://doi. org/10.1080/713688546

Harris, D. N. y Sass, T. R. (2011). Teacher training, teacher quality and student achievement. Journal of Public Economics, 95(7-8), 798-812. https:// doi.org/10.1016/j.jpubeco.2010.11.009

Hernández-Pino, U. (2005). Propuesta Curricular para la consolidación de los Semilleros de Investigación como espacios de Formación Temprana en Investigación. Revista Electrónica de la Red de Investigación Educativa 1(2).
Instituto Tecnológico de Estudios Superiores de Monterrey (ITESM). Aprendizaje basado en investigación. Investigación e Innovación Educativa. Recuperado el 20 de noviembre del 2018 en https://cutt.ly/8h651NI

Ion, G. (2014). Teachers as research promoters. In Schratz,M, Pecek, M \& Iucu, R. (eds) The changing role of teachers. European network on teacher education policies, Bucharest, Ars Docendi, (pp. 196-218).

Korthagen, F.A. (2010). La práctica, la teoría y la persona en la formación del profesorado. Revista Interuniversitaria de Formación del Profesorado, (68), 83-102.

Lampert, M. (2009). Learning teaching in, from, and for practice: What do we mean? Journal of Teacher Education 61(1-2) 21-34. https://doi. org/10.1177/0022487109347321

Larenas Díaz, C., Rodríguez Solar M. I., Soto Hernández, V., Conejero Solar, M., Vargas Morales, J. (2015). Temas clave en la formación de profesores en Chile desde la perspectiva de docentes y directivos. Revista Complutense de Educación, 26(3), 543.

Levy, P. y Petrulis, R. (2012). How do first-year university students experience inquiry and research, and what are the implications for the practice of inquiry-based learning? Studies in Higher Education, 37(1), 85-101.

Mansilla y Garrido (2019). Investigación educativa en las facultades de Educación en Chile: :"de", “en” o "con" los profesores? En Torres, B. (Comp.) Educación, escuela y profesorado: Aportes desde el Consejo de Decanos de Facultades de Educación del Consejo de Rectores de las Universidades Chilenas (CONFAUCE).

(pp. 13-29).Concepción: Editorial Universidad de Concepción.

Meerah, T., y Halim, L. (2012). Graduate information research skills. International Journal of Learning, 18(10), 91-100.

Ministerio de Educación de Chile (2016). Resultados Evaluación Docente 2015. Santiago: Centro de 
Perfeccionamiento, Experimentación e Investigaciones Pedagógicas (CPEIP). Recuperado de http://www.docentemas.cl/docs/Resultados_ Evauacion_Docente_2015.pdf

Murillo, F. J., Perines, H. y Lomba, L. (2017). La comunicación de la investigación educativa. Una aproximación a la relación entre la investigación, su difusión y la práctica docente. Profesorado, Revista de Curriculum y Formación del Profesorado, 21(3), 183-200.

Parra, C. (2004). Apuntes sobre la investigación formativa. Educación y Educadores, 7, 57-77.

Peña Vera, T. y Pirela Morillo, J. (2007). La complejidad del análisis documental. Información, Cultura y Sociedad, (16), 55-81.

Peñaherrera León, M., Chiluiza García, K. y Ortiz Colón, A. M. (2014). Inclusión del Aprendizaje Basado en Investigación ( $\mathrm{ABI}$ ) como práctica pedagógica en el diseño de programas de postgrados en Ecuador. Elaboración de una propuesta. Journal for Educators, Teachers and Trainers, 5(2), 204-220.

Perines, H. (2018). ¿Por qué la investigación educativa no impacta en la práctica docente? ESE: Estudios sobre Educación, (34), 9-27.

Perines, H. y Murillo F. J. (2017a). Percepciones de los docentes en formación sobre la investigación educativa. Estudios Pedagógicos, 43(1), 251-268. http://dx.doi.org/10.4067/S0718-07 052017000100015

Perines, H. y Murillo F. J. (2017b). ¿Cómo mejorar la investigación educativa? Sugerencias de los docentes. Revista de la Educación Superior, 46(181), 89-104. https://doi.org/10.1016/j.resu. 2016.11.003

Perines, H y Campaña, K. (2019). La "alfabetización” de los futuros docentes en investigación educativa: una reflexión teórica desde el contexto de Chile. RECIE, Revista Caribeña de Investigación Educativa, 3(1), 7-18. https://doi.org/10.32541/ recie.2019.v3i1.pp7-18

Perrenoud, P. (2007). Desarrollar la práctica reflexiva en el oficio de enseñar. Barcelona: GRAÓ.
Pirela, L. y Prieto, L. (2006). Perfil de competencias del docente en la función de investigador y su relación con la producción intelectual. Opción, 25(50). 159-177.

Rubio, M. J., Torrado, M., Quirós, C. y Valls, R. (2018). Autopercepción de las competencias investigativas en estudiantes de último curso de Pedagogía de la Universidad de Barcelona para desarrollar su Trabajo de Fin de Grado. Revista Complutense de Educación, 29(2), 335. https:// doi.org/10.5209/RCED.52443

Ruíz, C. y Torres, V. (2002). Actitud hacia el aprendizaje de la investigación: Conceptualización y medición. Educación y Ciencias Humanas, 10(18), 69-94.

Scherping, G. (2010). El caso de la formación de profesores. En Consejo Nacional de Educación/ Comisión Nacional de Acreditación. Seminario Internacional 2009. Calidad de los egresados, responsabilidad social ineludible. (pp. 175/178). Santiago: Andros Impresores.

Todd, M., Bannister, P. y Clegg, S. (2004). Independent inquiry and the undergraduate dissertation: Perceptions and experiences of final-year social science students. Assessment \& Evaluation in Higher Education, 29(3), 335-355. Doi: dx.doi. org/10.1080/0260293042000188285

Tovar-Gálvez, J. C. (2018). Formación investigativa de profesores: El problema como producto del proceso de problematización. Profesorado, Revista de Curriculum y Formación del Profesorado, 22(1), 133-148.

Valdés, A. A.; Vera, J. A.; y Carlos, E. A. (2012). Medición de competencias científicas en profesores de educación superior tecnológica. Avaliacao, 17(1). 237-254.

van der Linden, W., Bakx, A., Ros, A., Beijaard, D. y Vermeulen, M. (2012). Student teachers' development of a positive attitude towards research and research knowledge and skills. European Journal of Teacher Education, 35(4), 401-419.

van Dijk, T. (1980). Estructuras y funciones del discurso. México D. F.: Siglo Veintiuno. 
Vetter, P. y Ingrisani, D. (2013). Der Nutzen der forschungsmethodischen Ausbildung für angehende Lehrpersonen. Beiträge zur Lehrerinnen-und Lehrerbildung, 31(3), 321-332.

Zeichner, K. (1995). Beyond the divide of teacher research and academic research. Teachers and Teaching, 1(2), 153-172.
Zeichner, K. (2010). Rethinking the connections between campus courses and field experiences in college-and university-based teacher education. JournalofTeacherEducation, 61(1-2), 89-99. Doi: https://doi.org/10.1177/0022487109347671 